\title{
Self-efficacy and humanitarian aid workers
}

\author{
Christine R. Turner ${ }^{2}$, Donald Bosch ${ }^{1}$ and Anne A. T. Nolty ${ }^{1,2^{*}}$ (D)
}

\begin{abstract}
As a result of frequent exposure to trauma, aid workers are at high risk for negative psychological symptoms. Training specifically geared at fostering critical incident self-efficacy in humanitarian aid workers may bolster critical incident self-efficacy as well as general self-efficacy as they relate to experiences of traumatic symptomatology and resilience. Sixty-three aid workers completed questionnaires regarding efficacy, resilience, coping, and posttraumatic stress symptomatology at baseline, and 46 aid workers completed the same measures after the training workshop. Multiple regression analysis indicated that higher levels of self-efficacy related to higher resilience levels. General self-efficacy and critical incident coping self-efficacy (CICSE) were stronger after the training, even when controlling for histories of trauma. Histories of trauma contributed significant variance to CICSE before the training but were insignificant after the training. These findings suggest that aid organizations can support their workers by providing training that promotes resilience through enhancing efficacies.
\end{abstract}

Keywords: Resilience, General self-efficacy, Critical incident coping, Trauma, Humanitarians

\section{Introduction}

Aid workers experience continuous, episodic, personal, communal, and vicarious trauma. Many are resilient, overcoming traumatic symptomatology at least partially because they have a sense of self-efficacy, which is defined as the belief in one's ability to cope with traumatic situations (Benight \& Bandura, 2004). A sense of general self-efficacy enables people to interpret situations as challenging rather than stressful (Ebstrup et al., 2011), and yet, no researchers thus far have examined the value of self-efficacy to help humanitarian aid workers become more resilient. Further, although self-efficacy is known to help with the transfer of training (Stanhope et al., 2013), researchers have yet to explore its role within this population.

\section{Humanitarian aid workers and stress}

Humanitarian aid workers are front-line workers, susceptible to the impact of personal or vicarious critical incidents (Connorton et al., 2012). Some aid workers reside in war torn areas with threats of bombs, targeted

\footnotetext{
* Correspondence: annenolty@fuller.edu

${ }^{1}$ Headington Institute, Pasadena, CA, USA

${ }^{2}$ Graduate School of Psychology, Fuller Theological Seminary, Pasadena, CA, USA
}

attacks, and kidnappings, and others help in natural disasters, where cultural and physical isolation can contribute to their vulnerability (McFarlane, 2004), as can separation from their family (Connorton et al., 2012). In structure, policies, and practices of humanitarian opera(Strohmeier \& Panterick navigating what is often a "masculinized environment focusing on risk, danger, and the need for social separation in different forms" (Houldey, 2019, p.351). Sexual violence, which predominantly affects women, can be perpetrated within as well as without (Stoddard et al., 2019).

On a more subtle level, experiences for aid workers are transient and subjective, highly dependent on their gender, race, and country of origin (Strohmeier and and secondary trauma through their environment while enduring daily cumulative and traumatic episodic stress (Eriksson et al., 2001; McFarlane, 2004), those 
who have experienced previous trauma or have a history of mental illness are more likely to experience depressive and posttraumatic stress disorder (PTSD) symptoms as a result of future critical incidents (Eriksson et al., 2012). As a result of such experiences, many aid workers are more depressed or anxious or have more symptoms of posttraumatic stress, than members of the general population (Connorton et al., 2012). For aid workers, there is a strong correlation between reported hyperarousal symptoms and trauma due to actions of another human (Putman et al., 2009). Additionally, there is a high risk of burnout: $40 \%$ of aid workers in one study scored in the high-risk range of burnout subscales (Eriksson et al., 2009). These factors make aid workers susceptible to adverse symptoms, but also highlight opportunities to bolster aid workers. "Resilience humanitarianism (Hilhorst, 2018) links relief to development and focuses on local people and institutions as the first responders to crisis" (Hilhorst et al., 2019, p. S109). This shift in governance paradigm "requires investing in understanding, negotiating, and supporting national actors to responsibly implement humanitarian programming for vulnerable people" (p. S128).

Most aid workers are exposed to stress and potentially traumatic incidents, yet not every aid worker exposed to trauma reports adverse symptoms (Lopes Cardozo et al., 2013). In fact, Lopes Cardozo et al. (2013) noted that older aid workers reported less anxiety compared to their younger coworkers. In a study involving aid workers in Uganda, workers for the United Nations reported fewer mental health symptoms than those from smaller organizations: The organizational culture seems to impact the experience of the aid worker, such that more formalized structure and staff rapport seem to contribute to fewer adverse symptoms (Ager et al., 2012).

Resilience describes the process in which someone experiences a critical incident, perhaps temporarily passing through grief and sadness, but then coming back to his or her baseline of functioning, whereas a person who is not as resilient remains symptomatic for a longer period of time and struggles to return to normal functioning (Bonanno \& Mancini, 2012). A resilient person experiences the potentially traumatic experience and then adapts (Luthar et al., 2000). Resilience is thus an adaptive and interactive construct, wherein, by intervening, a person can decrease or increase his or her personal resilience (Herrman et al., 2011). The Headington Institute, an organization that supports aid workers through research and hands-on care, has identified seven individual factors that contribute to resilience: adaptive engagement, spirituality, emotional regulation and cognitive clarity, behavioral regulation, physical fitness, sense of purpose, and life satisfaction (Nolty et al., 2018).

Perceived organizational support is also important, as it buffers the negative impact of trauma; however, there is often a perceived lack of support for the workers (Connorton et al., 2012). In a study regarding local aid workers in Sri Lanka, aid workers who had less organizational support reported higher levels of depression and more posttraumatic stress symptoms (Lopes Cardozo et al., 2013). In addition, aid workers who report more social support are less likely to report burnout symptoms and more likely to report greater life satisfaction (Eriksson et al., 2012). Thus, policy decisions that can improve resilience for humanitarian workers must attend to power dynamics (Panter-Brick, 2021) as they focus on better quality work situations and more interconnected teams (Strohmeier \& Panter-Brick, 2020).

\section{Self-efficacy}

Self-efficacy refers to how a person perceives his or her ability to do what is necessary in a demanding situation (Bandura, 1982). According to Bandura (1982), past experiences, verbal persuasion, and physiological arousal influence a person's perception of his or her self-efficacy. Perceived self-efficacy reflects a specific domain, such as when one believes one has the ability to solve math problems or play sports, and is therefore measured with taskoriented questions (Luszczynska et al., 2005). In contrast, general self-efficacy reflects an overall sense of selfefficacy across all domains (Ebstrup et al., 2011). A sense of general self-efficacy is especially important for novel situations that require continual adaptability (Scholz et al., 2002) and that require efficacy across many domains (Ebstrup et al., 2011; Luszczynska et al., 2005)

The general self-efficacy measure (GSE), first developed in Germany (Schwarzer \& Jerusalem, 1995), is now available in 33 languages. In a meta-analysis of the GSE in 25 countries with 19,120 participants, Scholz et al. (2002) argued that general self-efficacy can be used as a universal construct across different cultures, as was suggested by Schwarzer and Born in 1997. The GSE yielded internal consistencies of .86 for the entire sample, with internal consistencies for each of the 25 countries ranging from $\alpha=.75$ to $\alpha=.91$ (Scholz et al., 2002).

\section{Self-efficacy and stress}

Self-efficacy is a fluid and protective construct that empowers people to act within traumatic contexts (Benight \& Bandura, 2004). Perceptions of self-efficacy are continually influenced by personal and secondary experiences, as well as physiological arousal (Bandura et al., 1977). One's sense of self-efficacy lessens due to a focus on deficits, strangeness, and struggles in a new situation and comparing one's self to others (Bandura, 1982). On the other hand, one's perception of self-efficacy strengthens through mastery learning, personal accomplishments, and interventions targeted at lowering emotional and physiological arousal (Bandura, 1982). 
Self-efficacy impacts the cognitive appraisal of stressful situations such that a person with stronger general selfefficacy tends to perceive certain stimuli as challenging rather than stressful (Bandura, 1997; Ebstrup et al., 2011). People with high self-efficacy are better able to regulate their emotions and seek out social support to help cope with stressful situations, resulting in strategies that help regulate emotions (Bandura, 1997). A person with a stronger sense of self-efficacy should be less distressed by threats and more able to proactively engage the threat, thus reducing the experience of stress (Bandura, 1997).

\section{Self-efficacy and transfer of training}

There is a plethora of research on transfer of training, which is the term used to describe the impartation of skills and knowledge and their subsequent implementation into trainees' work, primarily within a business context (e.g., Sitzmann \& Ely, 2011). One's perception of self-efficacy impacts the effectiveness of transfer of training (Stanhope et al., 2013) as well as one's cognitive ability, motivation, and perceived utility of training (Grossman \& Salas, 2011). Self-efficacy, persistence, effort, and the level of the goals that are set seem to have the strongest effects on the transfer of training (Sitzmann \& Ely, 2011). A high level of self-efficacy strengthens the transfer of training in learning the work culture (Simosi, 2012).

People with a higher sense of self-efficacy are more motivated to learn the training material presented (Grossman \& Salas, 2011), especially when they see the relevance of the training and the connection between their effort in learning the skills or material and desired results (Chiaburu \& Lindsay, 2008). Additionally, selfefficacy is related to behavior change: The stronger the sense of self-efficacy, the more likely a person is going to change and incorporate the new learned behavior (Bandura, 1982).

\section{The gap}

Researchers have studied self-efficacy in the context of business (e.g., Sitzmann \& Ely, 2011), phobias (Bandura, 1982), firefighting (Lambert et al., 2012), natural disasters (Benight \& Harper, 2002; Benight et al., 1999; Pooley et al., 2013), motor vehicle accidents (Benight et al., 2008), bereavement from cancer (Benight et al., 2001), patients with chronic conditions (Leppin et al., 2014), and domestic violence (Benight et al., 2004). However, to date, no researchers have examined self-efficacy specifically with humanitarian aid workers who face ongoing threats of critical incidents. Likewise, although self-efficacy helps transfer training (Grossman \& Salas, 2011; Sitzmann \& Ely, 2011) and coping with exposure to potentially traumatic events (Benight \& Bandura, 2004), research about supporting humanitarian aid workers in training by targeting their sense of self-efficacy has not been done.

It is the purpose of this study to investigate the relationship of self-efficacy to resilience and stress, as well as between self-efficacy and training transfer. We hypothesized:

1. Stronger levels of self-efficacy would be related to a greater sense of overall resilience, less impact of stress, decreased traumatic symptoms, and more stress-reducing behaviors in aid workers.

2. Levels of self-efficacy would be stronger after a training seminar designed to bolster self-efficacy.

\section{Method}

\section{Participants}

Aid workers enrolled in three hostile environment awareness training (HEAT) events were recruited for this longitudinal study. This training seminar is a form of high-fidelity stress exposure training (HFSET). The model involves an integration of principles from the fields of psychology and security. Prior to attending all participants are screened by a psychologist utilizing a test battery tailored to the rigors of the deployments they will undertake. The training is a 5-day event that begins with classroom instruction on understanding the human stress response and security procedures. Simulations of typical stressor events are gradually introduced allowing participants to practice their stress reduction techniques and security awareness. The final day typically involves more intense stressors such as an illegal checkpoint. Throughout the training a psychologist is monitoring participants and actively engaging them. After the training, the psychologist contacts participants who may have struggled to ensure their well-being. HFSET is well recognized in various fields that require high performance and high demand in risk situations such as police, fire, aviation, and emergency medicine. These trainings are designed to help participants develop confidence and self-efficacy to face the psychological and physical stressors they experience in these professions. In order to participate in the workshops, English proficiency was required.

Prior to these three training workshops, 63 aid workers (25 females, 38 males) from 27 countries (18 Africa, 22 Asia, 14 Europe or North America, 8 Australia or New Zealand) completed questionnaires specific to this project. Ethnicities were not collected. Participants had an average of 42 years of age $(S D=10.5$, range $25-74)$, between 0 and 35 years of field experience $(M=11.1, S D=$ $8.29)$, and an average of 17 years of education $(S D=$ 2.1). Forty-eight were married or in a committed relationship, 3 were divorced or separated, and 12 were single. The participants are employed by international non- 
governmental aid organizations who are preparing to be deployed to medium to high-risk locations such as Somalia, Afghanistan, and South Sudan. Many are working in refugee camps in these areas where the risk of kidnapping and other violence is prevalent. In recent years, due to several high-profile legal cases involving duty of care, many international non-governmental aid organizations are requiring this type of training to prepare the staff who choose to take on these assignments.

The majority of these participants also completed questionnaires 3 months following the training workshops (48 out of 63). There was no significant difference in age, education, or length of time as an aid worker between the 48 individuals ( 20 women, 28 men) who completed questionnaires 3 months following the workshops, and the 15 (5 women, 10 men) who did not.

\section{Measures \\ Resilience}

The Headington Institute Resilience Inventory (HIRI; Nolty et al., 2018) is a 38-item inventory that has a multifactor approach to resilience. The HIRI assesses seven factors: adaptive engagement (e.g., "I enjoy experimentation"), spirituality (e.g., "My life is enriched by my spiritual beliefs, practices and/or experiences"), emotional regulation (e.g., "My emotions are unmanageable"), behavioral regulation (e.g., "I finish what I start"), physical fitness (e.g., "I am unable to stick to an exercise program"), sense of purpose (e.g., "I find meaning in my work"), and life satisfaction (e.g., "I find satisfaction in life's small pleasures"). Respondents rate each item in regard to how accurately the item describes them, from 1 (not at all) to 7 (very well). For the individual factors at time 1, the $\alpha$ s in this study were .45 (physical fitness), .57 (sense of purpose), .63 (emotional regulation), .65 (life satisfaction), .70 (spirituality), .71 (behavioral regulation), and .82 (adaptive engagement). At time 2, they were generally stronger, ranging between .30 (physical fitness), .68 (life satisfaction), .72 (sense of purpose), .78 (spirituality), .80 (emotional regulation), .83 (adaptive engagement), and .86 (behavioral regulation). Alpha for the total HIRI score was .69 at time 1 and .67 at time 2 .

\section{Stress-reducing behaviors}

The Stress Vulnerability Scale (SVS; Miller \& Smith, 1985) is a 20 -item self-rated measure used to quantify respondents' engagement in activities that correlate with resilience, such as exercising regularly, engaging with social support, and eating a balanced diet. Respondents rate each item in regards to frequency of their participation from 1 (always) to 5 (never). The summation of the ratings (minus 20) suggests the level of respondents' vulnerability to stress. A final score over 50 indicates serious vulnerability to stress. A total score between 30 and 50 indicates vulnerability to stress, and a total score below 10 indicates excellent resistance to stress. In this study, at time $1, \alpha=$ .82 , and at time $2, \alpha=.77$.

\section{Traumatic symptoms}

The Posttraumatic Stress Disorder Checklist, Version 5 (PCL-5) is a 20-item self-report measure used to screen for PTSD, provide provisional PTSD diagnoses, and monitor symptoms over time. Each question identifies a symptom of PTSD in accordance with the Diagnostic and Statistical Manual of Mental Disorders (5th ed.; DSM-5; American Psychiatric Association, 2013). Respondents are asked to rate on a scale from 0 (not at all) to 4 (extremely) how much they have been bothered by each symptom in the past month. Scores theoretically range from 0 to 80 with higher scores reflecting more PTSD symptomatology. A decrease of five points or an increase of 10 points between administrations is considered a shift not due to chance, and a decrease of 10 points or an increase of 20 points is considered a clinically significant change in PTSD symptomatology (Weathers et al., 2013). In this study, the PCL-5 was used to gather clinical information about participants' experiences of PTSD symptomatology and to monitor PTSD symptom changes before and after the HEAT. Alpha was .94 at time 1 and .92 at time 2 .

\section{Impact of current stress}

Respondents were also asked to rate how much they have been "set back" by stress, on a scale from 0 to 10 , where higher scores reflect a greater impact of stress. This item was used as a global assessment of the respondent's perceived stress from the past week (Sheehan, 1990). Test-retest reliability has been estimated to be acceptable $(r=.86)$, and there is convincing evidence for validity (e.g., Connor et al., 2007). In a general U.S. population, Connor et al. (2007) found a mean score of $3.82(S D=3.16)$.

\section{General self-efficacy}

The GSE (Schwarzer \& Jerusalem, 1995) is a 10-item self-report measure to assess perceived self-efficacy in coping with daily life as well as stressful events. Participants indicate how well the statements describe them on a scale from 1 (not at all true) to 4 (exactly true). The composite scores range theoretically from 10 to 40 . In this study, scores ranged from 20 to 40 at time 1 , and from 25 to 40 at time 2. Alpha was .86 at time 1 and .90 at time 2 .

\section{Critical incident coping self-efficacy}

Benight et al. (1999) coping self-efficacy measure has been used in a variety of specific contexts. For this particular study, Benight gave permission to utilize the 
coping self-efficacy measure as a critical incident coping self-efficacy measure (CICSE). Respondents rated 9 items as to how capable they think they would be in dealing with a potential hostile critical incident. The scale was from 1 (not at all capable) to 7 (totally capable), with a theoretical range from 9 to 63. In this study, scores ranged from 32 to 63 at time 1 and from 25 to 63 at time 2. Alpha was .91 at time 1 and .94 at time 2 .

\section{Procedures}

This study used a combination of archival and newly collected data. The participants had previously completed most of the measures via Survey Monkey as a clinical requirement for their participation in the HEAT events (time 1). All individuals who planned to attend the training were also sent a link to a Survey Monkey questionnaire via email inviting them to complete an additional measure if they wished to participate in this research study. Three months after the training, individuals who had completed all the research measures were sent a link by email in order to complete all the measures again via Survey Monkey (time 2). The participants were not required to participate in this study in order to receive the HEAT.

HEAT is a form of high-fidelity stress exposure training or stress inoculation training. The idea of exposing individuals to stressors as a way of strengthening them for future exposures has a long history in medicine, psychology, sports, and the military. Given the hostile environments that humanitarians now deploy into, prior exposure to the potential dangers can help them be better prepared to respond appropriately. Psychological and physiological activation of the stress response is required in order for the individual to learn about their "stress signature" and engage coping mechanisms that can then be observed and improved upon through training. The opportunity to practice the management of stress responses under psychological supervision leads to greater preparedness, self-efficacy, and resilience when future stressors are encountered.

\section{Data analytic strategy}

To investigate hypothesis 1, Pearson's correlations revealed the relationship between participants' perceptions of general self-efficacy (GSE) and their perceived capacity to respond appropriately to potential critical incidents (CICSE) with the following study variables at time 1: HIRI, Sheehan, PCL-5, and SVS. Covariates included demographic variables, based on significant Pearson's correlations with the study variables. A hierarchal multiple regression demonstrated the determinants of the variance in self-efficacy. Post hoc tests elucidated which aspects of the HIRI were driving the correlations with self-efficacy.
For hypothesis 2, paired $t$ tests provided information to determine if there was a significant difference between time 1 GSE scores and time 2 GSE scores, and between time 1 CICSE scores and time 2 CICSE scores.

Because of the relatively small sample size for this study, the study was more susceptible to type II errors. Therefore, statistical trends were reported in order to present findings that might reasonably be expected in a study of higher power.

\section{Results}

\section{Descriptive analyses}

More than three quarters of the participants at time 1 reported a history of trauma (76\%): The 10 traumas that participants reported most frequently are presented in Table 1. HIRI total scores ranged from 32.7 to 47.8 ( $M=$ $41.1, S D=3.60)$ and SVS scores ranged from 21 to 64 $(M=17.5, S D=9.41)$. Total PCL-5 scores ranged from 0 to $40(M=9.3, S D=10.96)$ and Sheehan ratings ranged from 0 to 9 with a mode of 2 .

There was a significant positive correlation between GSE and CICSE scores at time $1, r(61)=.282, p=.025$. Controlling for participants' history of trauma made the correlation marginally stronger, $r(60)=.301 p=.017$, whereas controlling for other demographic variables made less difference.

We split the file by history of trauma in order to run a hierarchal regression analysis to further understand the relationship between GSE and CICSE. Variables that correlated at zero order with GSE and CICSE (years as an aid worker, Sheehan ratings, years of education, HIRI scores, and SVS scores) were entered at step 1. For participants with a history of trauma, the addition of the GSE in step 2 significantly increased the explanation of variance of CICSE, $\Delta R^{2}=.10, F(1,41)=6.76, p=.013$. However, for participants who reported no history of trauma, GSE did not significantly increase the explanation of the variance in CICSE at step $2, \Delta R^{2}=.02$, $F_{\text {change }}(1,7)=0.25, p=.63$.

\section{Hypothesis 1: Is self-efficacy correlated with HIRI, Sheehan, PCL-5, or SVS scores?}

Table 2 provides zero-order correlations for the GSE and CICSE with key study variables and demographics.

\section{GSE}

Participants' perceptions of general self-efficacy at time 1 correlated significantly with HIRI levels, $r(61)=.29, p$ $=.02$. However, participants' perceptions of general selfefficacy at time 1 were not significantly related to SVS, PCL-5, or Sheehan scores (see Table 2).

A hierarchal regression was used to further clarify the relative effects of demographic variables (years as an aid 
Table 1 Ten traumas reported most frequently

\begin{tabular}{llll}
\hline Type of trauma & $\mathbf{n}$ & $\mathbf{\%}^{\mathbf{a}}$ & $\mathbf{\%}^{\mathbf{b}}$ \\
\hline Being near gunfire & 17 & 27.0 & 25.4 \\
Being threatened with serious physical harm by someone & 16 & 23.8 & 33.4 \\
Picked up, arrested, taken away by soldiers or police & 15 & 22.2 & 31.3 \\
Being robbed or having your home broken into & 14 & 22.2 & 29.2 \\
Death of someone close (e.g., heart attack, car accident) & 14 & 20.6 & 29.2 \\
Being stopped at an illegal checkpoint & 13 & 19.0 & 27.1 \\
Other life-threatening event (e.g., natural disaster) & 12 & 17.5 & 25.0 \\
Being involved in an accident with serious injuries & 11 & 15.9 & 15.9
\end{tabular}

Note: ${ }^{\mathrm{a}}$ Percent of all participants $(N=63) .{ }^{\mathrm{b}}$ Percent of those who reported trauma $(n=48)$

worker and years of education) and of SVS, PCL-5, and Sheehan scores on GSE scores at time 1.

The number of years of education or as an aid worker did not significantly explain the variance in GSE scores at time 1 as can be seen in step 2 of Table 3, although there was a trend towards education as an explanation of variance for GSE scores, $\Delta R^{2}=.04, F_{\text {change }}(1,59)=2.84, p=.07$. Although the addition of SVS did not significantly improve the model, $\Delta R^{2}$ $=.03, F_{\text {change }}(1,58)=1.7, p=.20$, the addition of the HIRI contributed weakly to the variance associated with GSE scores at time $1, \Delta R^{2}=.04, F_{\text {change }}(1,57)=2.87, p=.10$.

\section{CICSE}

CICSE scores at time 1 and HIRI levels of resilience were correlated in a positive direction, $r(61)=.48, p<.001$. CICSE scores at time 1 were significantly negatively correlated with participants' perceptions of setback due to stress, $r(61)=-.38, p=.002$, as well as with PCL-5 scores, $r(61)=-.30, p=.02$, and SVS scores, $r(60)=-.26, p=.04$.

Table 4 provides the hierarchal regression used to further understand how these variables contributed to the explanation of variance of CICSE at time 1. Whether or not participants had a history of trauma explained some of the variance for CICSE at Time $1, \Delta R^{2}=.06, F_{\text {change }}$ $(1,60)=4.08, p=.05$, as did their Sheehan rating of how much they had been set back by stress in the previous week, $\Delta R^{2}=.07, F_{\text {change }}(1,58)=4.89, p=.031$. With the addition of the HIRI in step 5 , explained variance in scores of CICSE at Time 1 increased significantly, $\Delta R^{2}=$ $.11, F_{\text {change }}(1,56)=8.22, p=.006$, as the HIRI contributed a significant amount of the accounted variance of the model, $\beta=.38, t=2.87, p=.006$.

Table 2 Correlations between key study variables and demographic variables at time 1

\begin{tabular}{|c|c|c|c|c|c|c|c|c|c|c|c|c|}
\hline & $M(S D)$ & GSE & HIRI & SVS & PCL-5 & Sheehan & Age & Gender & Educ. & $\begin{array}{l}\text { Years as } \\
\text { worker }\end{array}$ & $\begin{array}{l}\text { Trauma } \\
\text { history }\end{array}$ & $\begin{array}{l}\text { Types of } \\
\text { trauma }\end{array}$ \\
\hline CICSE & $51.2(7.80)$ & $.28^{*}$ & $.48^{* *}$ & $-.26^{*}$ & $-.30^{*}$ & $-.39 * *$ & .02 & .01 & -.15 & .17 & $-.29^{*}$ & -.03 \\
\hline GSE & $31.6(4.44)$ & & $.29^{*}$ & -.22 & -.08 & -.07 & .12 & .01 & .24 & .18 & .02 & .15 \\
\hline $\mathrm{HIRI}$ & $41.1(3.60)$ & & & $-.46^{* *}$ & $-.29^{*}$ & $-.44^{* *}$ & .02 & .14 & -.05 & .20 & -.10 & .03 \\
\hline SVS & $17.5(9.41)$ & & & & $.41^{* *}$ & $.43^{* *}$ & .03 & -.23 & -.22 & -.10 & .16 & .04 \\
\hline$P C L-5$ & $9.3(10.96)$ & & & & & $.59^{* *}$ & .02 & -.24 & .07 & .11 & $.26^{*}$ & .16 \\
\hline Sheehan & $2.9(2.38)$ & & & & & & -.01 & -.22 & -.01 & -.02 & .18 & .04 \\
\hline Age & $42.4(10.51)$ & & & & & & & .01 & .10 & $.55^{* *}$ & .19 & .16 \\
\hline Gender & ------ & & & & & & & & .18 & -.05 & -.23 & -.05 \\
\hline Education & $17.1(2.07)$ & & & & & & & & & .15 & .08 & .10 \\
\hline Years as worker & $11.1(8.29)$ & & & & & & & & & & .20 & $.32^{*}$ \\
\hline $\begin{array}{l}\text { History of } \\
\text { trauma }\end{array}$ & $\begin{array}{l}48 \text { (yes) } 15 \\
\text { (no) }\end{array}$ & & & & & & & & & & & $.53^{* *}$ \\
\hline $\begin{array}{l}\text { \# types of } \\
\text { trauma }\end{array}$ & $2.9(3.08)$ & & & & & & & & & & & \\
\hline
\end{tabular}

Note. $N=61-63$. HIRI Headington Institute Resilience Inventory, SVS Stress Vulnerability Scale, PCL-5 Posttraumatic Stress Disorder Checklist Version 5, Sheehan Sheehan Global Assessment, CICSE critical incident coping self-efficacy, GSE general self-efficacy. History of trauma is a dichotomous variable. ${ }^{* *} p<.01 .{ }^{*} p<.05$ 
Table 3 Multiple regression analysis for general self-efficacy at time 1

\begin{tabular}{|c|c|c|c|c|c|}
\hline & $B$ & SE B & $\beta$ & $\Delta R^{2}$ & $p$ \\
\hline Step 1 & & & & .04 & .15 \\
\hline Years as an aid worker & 0.10 & 0.07 & 0.19 & & \\
\hline Step 2 & & & & .05 & .07 \\
\hline Years as an aid worker & 0.08 & 0.07 & 0.15 & & \\
\hline Years of education & 0.50 & 0.27 & 0.23 & & \\
\hline Step 3 & & & & .03 & .20 \\
\hline Years as an aid worker & 0.08 & 0.07 & 0.14 & & \\
\hline Years of education & 0.42 & 0.27 & 0.20 & & \\
\hline Stress Vulnerability & -0.08 & 0.06 & -0.17 & & \\
\hline Step 4 & & & & .04 & .10 \\
\hline Years as an aid worker & 0.05 & 0.07 & 0.10 & & \\
\hline Years of education & 0.51 & 0.27 & 0.24 & & \\
\hline Stress Vulnerability & -0.02 & 0.07 & -0.05 & & \\
\hline HIRI & 0.30 & 0.18 & 0.24 & & \\
\hline
\end{tabular}

Note. HIRI Headington Institute Resilience Inventory

Table 4 Multiple regression analysis for critical incident coping self-efficacy at time 1

\begin{tabular}{|c|c|c|c|c|c|}
\hline & $B$ & $S E B$ & $\beta$ & $\Delta R^{2}$ & $p$ \\
\hline Step 1 & & & & $.06^{*}$ & .05 \\
\hline History of Trauma & -4.67 & 2.31 & $-0.25^{*}$ & & \\
\hline Step 2 & & & & .05 & .07 \\
\hline History of Trauma & -3.40 & 2.30 & -0.22 & & \\
\hline Stress Vulnerability & -0.19 & 0.10 & -0.23 & & \\
\hline Step 3 & & & & $.07^{*}$ & .03 \\
\hline History of Trauma & -3.56 & 2.23 & -0.19 & & \\
\hline Stress Vulnerability & -0.09 & 0.11 & -0.10 & & \\
\hline Sheehan & -0.96 & 0.43 & $-0.29^{*}$ & & \\
\hline Step 4 & & & & .001 & .79 \\
\hline History of Trauma & -3.45 & 2.29 & -0.19 & & \\
\hline Stress Vulnerability & -0.08 & 0.11 & -0.10 & & \\
\hline Sheehan & -0.90 & 0.50 & -0.27 & & \\
\hline PCL-5 & -0.03 & 0.11 & -0.04 & & \\
\hline Step 5 & & & & $.11^{* *}$ & .01 \\
\hline History of trauma & -3.63 & 2.16 & -0.20 & & \\
\hline Stress Vulnerability & 0.03 & 0.11 & 0.04 & & \\
\hline Sheehan & -0.53 & 0.49 & -0.16 & & \\
\hline PCL-5 & -0.04 & 0.10 & -0.05 & & \\
\hline $\mathrm{HIRI}$ & 0.83 & 0.29 & $0.38^{*}$ & & \\
\hline
\end{tabular}

${ }^{*} p<.05,{ }^{* *} p<.01$

\section{CICSE and HIRI}

Post hoc correlations revealed that at time 1, the CICSE was positively correlated with adaptive engagement, $r(61)=.38, p=.002$, emotional regulation, $r(61)=.33, p$ $=.009$, behavioral regulation $r(61)=.60, p<.0001$, sense of purpose $r(61)=.37, p=.003$, and life satisfaction, $r(61)=.49, p<.0001$. The GSE, on the other hand, only correlated with adaptive engagement, $r(61)=.42, p=$ .001 , and behavioral regulation, $r(61)=.31, p=.015$. Controlling for the years as an aid worker, which appeared to trend toward a very weak correlation with total HIRI scores, $r(61)=.20, p=.122$, did not significantly alter the correlations between the self-efficacy variables and the HIRI factors (see Table 5).

\section{Hypothesis 2: Are there gains in GSE or CICSE scores following training?}

There was a significant difference in scores between time 1 GSE $(M=32.0, S D=4.36)$ and time 2 GSE $(M=33.4$, $S D=4.36), t(46)=2.08, p=.043$. When examining only participants with a history of trauma, there still was a significant difference in time 1 GSE $(M=32.3, S D=$ 4.05) and time $2 \operatorname{GSE}(M=33.7, S D=4.39), t(37)=$ $2.09, p=.044$.

The average CICSE score at time 1 was $51.43(S D=$ 7.88), and the average CICSE score at time 2, after training, was 51.54 $(S D=9.06), t(45)=.27, p=.79$. Findings were similar for the subgroup of participants with a history of trauma, between time $1 \mathrm{CICSE}(M=50.7, S D=$ $8.2)$ and time $2 \operatorname{CICSE}(M=51.3, S D=9.12), t(37)=$ $0.45, p=.65$.

\section{Are GSE scores related to gains in CICSE scores following training?}

There was a trend toward a significant correlation between time 1 GSE and time 2 CICSE, $r(44)=.29, p=$ .054 , which was not as strong when controlling for time 1 CICSE, $r(43)=.20, p=.192$.

Table 5 Correlations for GSE and CICSE at time 1 with HIRI factors, controlling for years as an aid worker

\begin{tabular}{llll}
\hline & $\boldsymbol{M}(\boldsymbol{S D})$ & CICSE & GSE \\
\hline Zero order & & & \\
Adaptive engagement & $5.9(0.76)$ & $.34^{* *}$ & $.42^{* *}$ \\
Spirituality & $5.8(1.27)$ & .01 & .17 \\
Emotional regulation & $5.7(0.84)$ & $.33^{* *}$ & .04 \\
Behavioral regulation & $5.9(0.72)$ & $.57^{* *}$ & $.31^{*}$ \\
Physical fitness & $5.3(1.05)$ & .17 & -.18 \\
Sense of purpose & $6.3(0.67)$ & $.37^{* *}$ & .23 \\
Life satisfaction & $6.2(0.64)$ & $.49^{* *}$ & .22 \\
\hline
\end{tabular}

Note. CICSE critical incident coping self-efficacy, GSE general self-efficacy, HIRI Headington Institute Resilience Inventory

${ }^{* *} p<.01,{ }^{*} p<.05$ 
To further examine the relationship, a hierarchal regression analysis was conducted with time 2 CICSE as the criterion variable (see Table 6). Time 1 CICSE was entered at step 1 and time 1 GSE was entered at step 2. Time 1 GSE tended toward a significant explanation of variance for time 2 CICSE, $\Delta R^{2}=.04, F_{\text {change }}(1,45)=$ $2.25, p=.141$.

An additional hierarchical regression analysis is presented in Table 7 with additional variables included. Time 1 CICSE was entered at step 1 , followed by years as an aid worker and history of trauma at step 2. Time 1 GSE was entered at step $3, \Delta R^{2}=.032, F_{\text {change }}(1,43)=2.01, p=.16$, with $\beta=.189, t(42)=1.42, p=.164$. Whether or not they had a history of trauma did not contribute significantly to the amount of variance in step 3.

At time 2, the history of trauma was no longer significantly related to CICSE scores or to any of the key study variables (see Table 8). The correlation between the CICSE and the GSE was stronger at time 2, $z=2.81, p=$ .003 , as was the correlation between the CICSE and the HIRI, $z=1.66, p=.049$. There was a weak trend toward a significantly stronger correlation between the CICSE and the PCL-5, $z=-1.15, p=.12$, but not between the CICSE and the SVS, $z=-0.347, p=.36$. The relationship between the GSE and the HIRI was stronger at time 2, $z=3.53, p=.000$, and there was a weak trend toward a stronger correlation between the GSE and the SVS, $z=$ $-1.17, p=.12$.

\section{Discussion}

One's sense of general self-efficacy is related to one's perception of being able to handle a critical incident

Generally, the more efficacious a person felt in their general ability to cope with life, the more efficacious they felt they would be to cope with a critical incident. Perceptions of general self-efficacy tended towards a relationship with perceptions of ability to cope with critical incidents when taking into consideration participants' history of trauma (i.e., controlling for history of trauma). However, when solely examining the relationship with those who reported no history of trauma, reports of general self-efficacy and coping with critical incident efficacy were not related. For participants who had experience with trauma, there was a significant relationship between

Table 6 Multiple regression analysis for time 2 CICSE

\begin{tabular}{|c|c|c|c|c|c|}
\hline & $B$ & $S E B$ & $\beta$ & $\Delta R^{2}$ & $p$ \\
\hline Step 1 & & & & .24 & .00 \\
\hline Time 1 CICSE & 0.55 & 0.15 & .47 & & \\
\hline Step 2 & & & & .04 & .14 \\
\hline Time 1 CICSE & 0.50 & 0.15 & .44 & & \\
\hline Time 1 GSE & 0.40 & 0.27 & .20 & & \\
\hline
\end{tabular}

Note. CICSE critical incident coping self-efficacy, GSE general self-efficacy
Table 7 Multiple regression analysis for time 2 CICSE accounting for history of trauma

\begin{tabular}{lccccc}
\hline & $\boldsymbol{B}$ & $\boldsymbol{S E} \boldsymbol{B}$ & $\boldsymbol{\beta}$ & $\boldsymbol{\Delta \boldsymbol { R } ^ { \mathbf { 2 } }}$ & $\boldsymbol{p}$ \\
\hline Step 1 & & & & .24 & .00 \\
$\quad$ Time 1 CICSE & 0.55 & 0.15 & .49 & & \\
Step 2 & & & & .04 & .32 \\
$\quad$ Time 1 CICSE & 0.62 & 0.15 & .54 & & \\
$\quad$ Years as an aid worker & 0.20 & 0.14 & .19 & & \\
$\quad$ History of trauma & 2.63 & 3.00 & .12 & & \\
Step 3 & & & & .03 & .16 \\
$\quad$ Time 1 CICSE & 0.56 & 0.16 & .49 & & \\
$\quad$ Years as an aid worker & 0.20 & 0.14 & .19 & & \\
$\quad$ History of trauma & 1.76 & 3.03 & .08 & & \\
$\quad$ Time 1 GSE & 0.39 & 0.27 & .19 & & \\
\hline Note. CICSE critical incident coping self-efficacy, GSE general self-efficacy &
\end{tabular}

Note. CICSE critical incident coping self-efficacy, GSE general self-efficacy

the two types of efficacies. Participants seemed to need some experience with trauma in order to have a realistic perspective on how efficaciously they could handle a critical incident. This finding is congruent with Bandura's (1982) theory that past experiences (i.e., in this case, trauma) influence a person's perception of self-efficacy.

\section{Hypothesis 1: Self-efficacy relates to resilience levels}

The data supported some aspects of hypothesis 1 . In regard to resilience, the more efficacious the participant, the higher the participant's resilience level. A stronger sense of general self-efficacy and of being able to cope with a critical incident was related to higher resilience, whether or not there had been a history of trauma. More specifically in terms of resilience, people who were more adaptive, more emotionally regulated, or more behaviorally regulated were more likely to report feeling more capable of coping with a critical incident. This finding is congruent with prior suggestions that people who are more efficacious utilize social supports and more effective coping strategies (e.g., Luszczynska et al., 2005).

As predicted, participants in this current study who reported feeling less set back from the stresses encountered the previous week reported higher levels of general self-efficacy, despite the fact that all participants may not have experienced the same degree of stressful events in the previous week. This association between being less set back from stresses and higher levels of general selfefficacy is possibly congruent with Bandura's (1997) understanding of people who are more efficacious not evaluating a difficult situation as distressing, but instead, as challenging (Ebstrup et al., 2011).

However, contrary to the study hypothesis, participants' stress-reducing behaviors did not appear to be related to a greater sense of general self-efficacy. This is not congruent with the research that has shown that 
Table $\mathbf{8}$ Correlations between key study variables at time 2

\begin{tabular}{lllllllrr}
\hline Variable & $\boldsymbol{N}$ & $\boldsymbol{M}(\mathbf{S D})$ & T2_GSE & T2_HIRI & T2_SVS & T2_PCL-5 & T2_Sheehan & Trauma History \\
\hline T2_CICSE & 48 & $51.54(9.06)$ & $.61^{* *}$ & $.65^{* *}$ & $-.31^{*}$ & $-.45^{* *}$ & $-.39^{* *}$ & -.05 \\
T2_GSE & 48 & $33.56(4.37)$ & & $.68^{* *}$ & $-.38^{* *}$ & -.23 & -.28 & -.07 \\
T2_HIRI & 50 & $39.94(3.92)$ & & & $-.51^{* *}$ & $-.31^{*}$ & $-.35^{*}$ & .06 \\
T2_SVS & 50 & $36.36(9.10)$ & & & & $.41^{* *}$ & .02 \\
T2_PCL-5 & 50 & $7.24(8.48)$ & & & & & $.52^{* *}$ \\
T2_Sheehan & 50 & $2.94(2.68)$ & & & & & -.25 \\
History of trauma & 63 & - & & & & \\
\hline
\end{tabular}

Note: T2_CICSE critical incident coping self-efficacy, T2_GSE general self-efficacy, T2_HIRI Headington Institute Resilience Inventory, T2_SVS Stress Vulnerability Scale, T2_PCL-5 Posttraumatic Stress Disorder Checklist Version 5, T2_Sheehan Sheehan Global Assessment, History of trauma is a dichotomous variable ${ }^{* *} p<.01 .{ }^{*} p<.05$

more efficacious people will more successfully implement positive health behaviors (Bandura, 1997). This study may be elucidating more salient features of those who feel they would be capable of coping with a critical incident, such as prior exposure to trauma and levels of resilience. However, the findings may also highlight the nature of an aid worker, which may not include similar positive health behaviors as those present in Bandura's research.

Levels of posttraumatic stress symptomatology were negatively related to levels of critical incident self-efficacy. The variance of critical incident self-efficacy was more aptly explained by other factors. After taking into account whether or not participants had a prior history of trauma, there was no longer a relationship between critical incident coping self-efficacy and trauma symptomatology. That is to say, experiences of trauma likely informed one's self-efficacy with regard to a critical incident, regardless of one's level of posttraumatic stress symptomology.

In this study, those without a history of prior trauma at time 1 were more likely to indicate that they felt efficacious to face a critical incident. Those who had never experienced trauma potentially demonstrated naiveté in their self-report of how confident they might feel in future hypothetical critical incidents. Therefore, though aid workers with a history of trauma reported feeling less efficacious, they may actually have a more realistic perception of their abilities because they have encountered trauma. Those who experienced trauma were also more likely to experience posttraumatic stress symptoms, such as intrusive thoughts, nightmares, avoiding emotions, feeling isolated, blame, negative belief's about self, increase arousal, and increased irritability (American Psychiatric Association, 2013). Clinically, it makes sense that participants reporting higher levels of posttraumatic symptoms may not feel efficacious in their ability to "deal with emotions," "not 'lose it' emotionally, " or "not be critical about the incident," as stated in CICSE items. The posttraumatic stress symptoms seem to address the loss of self-efficacy.
Hypothesis 2: Gains in one's sense of general self-efficacy but not for critical incidents

Participants' sense of general self-efficacy seemed to increase when they completed questionnaires 3 months after they had attended a training focused on preparing them for high-risk situations. The training provided tools intended to enhance participants' perception and ability to cope with diverse experiences, such as emotions, physiological arousal, critical thinking, and preventative health care. According to the results, the training seemed to enhance participants' sense of selfefficacy. This study builds upon the self-efficacy literature of transfer of training (e.g., Chiaburu \& Lindsay, 2008; Grossman \& Salas, 2011; Simosi, 2012) by showing the importance of training geared at self-efficacy for aid agencies. People with higher self-efficacy engage in training in a way that helps them feel confident to apply the knowledge and continue to boost their self-efficacy regarding the training.

There was not a significant increase in perceptions of ability to cope with critical incidents even when taking into account the participant's history of trauma. The training was geared toward teaching skills around coping with critical incidents through role-playing a critical incident. The participants therefore experienced a controlled critical incident, which likely provided a more realistic perspective on participants' ability to cope. Following the training, when asked how well they thought that they could handle a critical incident, it seems likely that they were able to respond more realistically than they had prior to the training.

One's general sense of self-efficacy may facilitate gains in one's sense of ability to cope with critical incidents

Because the goal of the training was to increase one's efficacy to cope with critical incidents, higher levels of the general sense of self-efficacy were expected to facilitate increase and implementation training knowledge (i.e., critical incident coping self-efficacy). The weak trend toward a significant correlation between participants' 
general self-efficacy at time 1 and an increase in critical incident coping self-efficacy at time 2 is congruent with suggestions from research that higher self-efficacy strengthens transfer of training (Simosi, 2012).

At time 2, participants with a higher sense of their ability to cope with critical incidents were likely to have stronger ratings of resilience and to report less experience of posttraumatic stress symptoms. Further, at time 2, one's history of trauma was not related to the other study variables. The experience of trauma did not impact their sense of efficacy. However, at time 1, one's history of trauma was significantly related to other study variables.

Because the relationships between CICSE and other variables were strongly correlated at time 2 , perhaps the time 2 CICSE was actually a more accurate measure of coping with critical incident self-efficacy. The naiveté of those with no trauma history may have impacted the accuracy of their perception of their ability to cope with critical incidents as previously proposed. Not only was there perhaps a more accurate reflection of coping self-efficacy in regard to critical incidents after the training, but also there did not seem to be a significant impact of prior experiences of trauma on the study variables. That is to say, prior to training, a history of trauma explained a lot of the variance in participants' scores, whereas after the training, trauma was not a major factor. Tools, and the chance for the participants to experience a sense of mastery, were provided at the training. Findings in the literature support the idea that opportunities for mastery learning enhance a person's sense of self-efficacy (Bandura, 1982).

\section{Limitations and suggestions for further research}

There are several limitations to this study. First, the data were gathered via the self-report of aid workers. Selfreport measures rely on the perception and understandings of the participant and do not allow opportunity for clarification from the researcher. Due to the nature of the questionnaire, the present study could not statistically address the differences in the impact of types of trauma, nor examine the impact of trauma on the field. In future research, it would be helpful to consider the impact of different types and intensities of trauma on one's perception of self-efficacy, with a particular focus on whether or not the trauma had occurred within the line of duty as an aid worker or in one's civilian life.

It would also be helpful to broaden the research to include aid workers and humanitarian volunteers from different agencies, comparing differences between agencies as well as between paid workers and volunteers. Comparisons in self-efficacy between national aid workers from high-risk environments in need of humanitarian aid versus expatriate aid workers from countries that are not in need of widespread humanitarian aid were not addressed, which was a limitation of the study. Similarly, it would be good to compare aid workers who have had minimal time on the field with seasoned aid workers, as it may take time to develop coping strategies, and even aid workers who have dropped out, perhaps because they were not able to develop coping strategies. Qualitative studies would be helpful.

Further, the construct of self-efficacy seems to be an important consideration for aid workers and should be further studied in the context of empowering aid workers. For example, it would be helpful to evaluate specific interventions that should help bolster selfefficacy in coping with critical incidents. In particular, each of the HIRI factors of resilience might provide an area to bolster through efficacy-enhancing interventions.

\section{Conclusion}

Aid workers voluntarily work in complex situations where they are exposed to various types of stress and critical incidents. This research suggests that the more efficacious a person feels, the more likely they will demonstrate resilience. Efficacy and resilience are both fluid constructs. In this study, training geared at helping aid workers cope with critical incidents seems to help bolster aid workers' sense of efficacy and, in turn, their level of resilience. Providing aid workers with a training that allowed them to gain a sense of mastery in critical incident seems to have helped reduce the negative impact of their previous trauma. Although there is a plethora of research and resources on the treatment for posttraumatic stress disorder (National Center for PTSD, 2016), this study differs in that it highlights preventative intervention that can support aid workers in their experience of trauma in the field. General self-efficacy and coping with critical incident self-efficacy are entry points to better support aid workers.

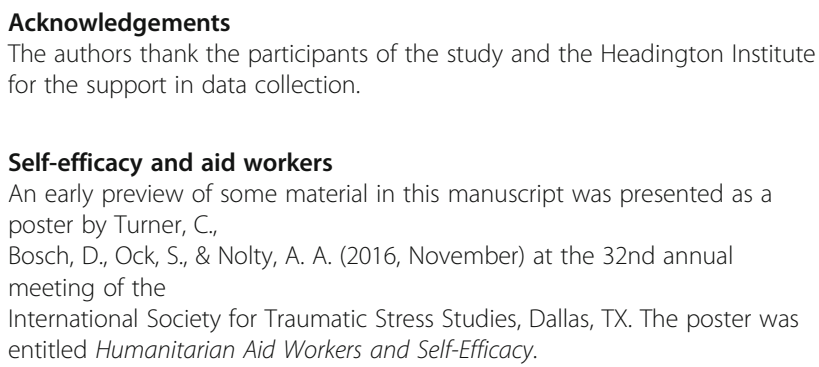

Self-efficacy and aid workers

An early preview of some material in this manuscript was presented as a poster by Turner, $\mathrm{C}$.

Bosch, D., Ock, S., \& Nolty, A. A. (2016, November) at the 32nd annual meeting of the

International Society for Traumatic Stress Studies, Dallas, TX. The poster was entitled Humanitarian Aid Workers and Self-Efficacy.

\section{Authors' contributions}

Each author materially participated in the research and/or article preparation. The authors' individual credit roles are as follows: C T: Conceptualization, data curation, formal analysis, methodology, writing - original draft. D B: Supervision, writing - review \& editing. A N: Conceptualization, formal analysis, supervision, writing-review \& editing. The authors read and approved the final manuscript. 


\section{Availability of data and materials}

Upon reasonable request.

\section{Competing interests}

The authors declare that they have no competing interests.

Received: 26 March 2020 Accepted: 19 February 2021

Published online: 06 March 2021

\section{References}

Ager A, Pasha E, Yu G, Duke T, Eriksson CB, Cardozo BL (2012) Stress, mental health, and burnout in national humanitarian aid workers in Gulu, Northern Uganda. J Traum Stress 25:713-720. https://doi.org/10.1002/jts.21764

American Psychiatric Association (ed) (2013) Diagnostic and statistical manual of mental disorders, 5th edn. American Psychiatric Association, Washington

Bandura A (1982) Self-efficacy mechanism in human agency. Am Psychol 37:122147. https://doi.org/10.1037/0003-066x.37.2.122

Bandura A (1997) Insights: self-efficacy. Harvard Mental Health Letter 13(9):4-6

Bandura A, Adams NE, Beyer J (1977) Cognitive processes mediating behavioral change. J Pers Soc Psychol 35:125-139. https://doi.org/10.1037/0022-3514.3 5.3.125

Benight CC, Bandura A (2004) Social cognitive theory of posttraumatic recovery: the role of perceived self-efficacy. Behav Res Ther 42:1129-1148. https://doi. org/10.1016/j.brat.2003.08.008

Benight CC, Cieslak R, Molton IR, Johnson LE (2008) Self-evaluative appraisals of coping capability and posttraumatic distress following motor vehicle accidents. J Consult Clin Psych 76:677-685. https://doi.org/10.1037/0022$006 x .76 .4 .677$

Benight CC, Flores J, Tashiro T (2001) Bereavement coping self-efficacy in cancer widows. Death Stud 25(2):97-125. https://doi.org/10.1080/074811801461900

Benight CC, Harding-Taylor AS, Midboe AM, Durham RL (2004) Development and psychometric validation of a domestic violence coping self-efficacy measure (DV-CSE). J Trauma Stress 17:505-508. https://doi.org/10.1007/s10960-0045799-3

Benight CC, Harper ML (2002) Coping self-efficacy perceptions as a mediator between acute stress response and long-term distress following natural disasters. J Trauma Stress 15:177-186. https://doi.org/10.1023/a101529502 5950

Benight CC, Ironson G, Durham RL (1999) Psychometric properties of a hurricane coping self-efficacy measure. J Trauma Stress 12:379-386. https://doi.org/10.1 023/a1024792913301

Bonanno GA, Mancini AD (2012) Beyond resilience and PTSD: mapping the heterogeneity of responses to potential trauma. Psychol Trauma-US 4:74-83. https://doi.org/10.1037/a0017829

Chiaburu DS, Lindsay DR (2008) Can do or will do? The importance of selfefficacy and instrumentality for training transfer. Hum Resour Dev Int 11:199206. https://doi.org/10.1080/13678860801933004

Connor KM, Vaishnavi S, Davidson JR, Sheehan DV, Sheehan KH (2007) Perceived stress in anxiety disorders and the general population: a study of the Sheehan Stress Vulnerability Scale. Psychiat Res 151:249-254. https://doi. org/10.1016/.jpsychres.2006.11.008

Connorton E, Perry MJ, Hemenway D, Miller M (2012) Humanitarian relief workers and trauma-related mental illness. Epidemiol Rev 34:145-155. https://doi. org/10.1093/epirev/mxr026

Ebstrup JF, Eplov LF, Pisinger C, Jørgensen T (2011) Association between the five factor personality traits and perceived stress: is the effect mediated by general self-efficacy? Anx Stress Copin 24:407-419. https://doi.org/10.1080/1 0615806.2010.540012

Eriksson CB, Bjorck JP, Larson LC, Walling SM, Trice GA, Fawcett J, Abernethy A, Foy DW (2009) Social support, organizational support, and religious support in relation to burnout in expatriate humanitarian aid workers. Ment Health Rel Cult 12:671-686. https://doi.org/10.1080/13674670903029146

Eriksson CB, Kemp HV, Gorsuch R, Hoke S, Foy DW (2001) Trauma exposure and PTSD symptoms in international relief and development personnel. J Trauma Stress 14:205-212. https://doi.org/10.1023/A1007804119319

Eriksson CB, Lopes Cardozo BL, Foy DW, Sabin M, Ager A, Snider L, Scholte WF, Kaiser R, Olff M, Rijnen B, Crawford CG, Zhu J, Simon W (2012) Predeployment mental health and trauma exposure of expatriate humanitarian aid workers: Risk and resilience factors. Traumatol 19:41-48. https://doi.org/10.1177/1534765612441978
Grossman R, Salas E (2011) The transfer of training: what really matters. Int J Train Dev 15:103-120. https://doi.org/10.1111/j.1468-2419.2011.00373.x

Herrman H, Stewart D, Diaz-Granadoz N, Berger E, Jackson B, Yuen T (2011) What is resilience? Can J Psychiat 56:258-265

Hilhorst D (2018) Classical humanitarianism and resilience humanitarianism: making sense of two brands of humanitarian action. J Int Humanit Action 3(15):1-12 https://doi.org/10.1186/s41018-018-0043-6

Hilhorst D, Desportes I, de Milliano CWJ (2019) Humanitarian governance and resilience building: Ethiopia in comparative perspective. Disasters 43:S109S131 https://doi.org.fuller.idm.oclc.org/10.1111/disa.12332

Houldey G (2019) Humanitarian response and stress in Kenya: gendered problems and their implications. Gend Dev 27(2):337-353 https://doi.org. fuller.idm.oclc.org/10.1080/13552074.2019.1615281

Lambert JE, Benight CC, Harrison E, Cieslak R (2012) The firefighter coping selfefficacy scale: measure development and validation. Anxiety Stress Coping 25(1):79-91. https://doi.org/10.1080/10615806.2011.567328

Leppin AL, Bora PR, Tilburt JC, Gionfriddo MR, Zeballos-Palacios C, Dulohery MM, Sood A, Erwin P, Brito JP, Boehmer KR, Montori VM (2014) The efficacy of resiliency training programs: a systematic review and meta-analysis of randomized trials. PloS One 9(10):e111420. https://doi.org/10.1371/journal. pone. 0111420

Lopes Cardozo B, Sivilli TI, Crawford C, Scholte WF, Petit P, Ghitis F, Ager A, Eriksson C (2013) Factors affecting mental health of local staff working in the Vanni region, Sri Lanka. Psychol Trauma-US 5:581. https://doi.org/10.1037/a 0030969

Luszczynska A, Scholz U, Schwarzer R (2005) The general self-efficacy scale: multicultural validation studies. J Psychol 139:439-457. doi:10 .3200/jlp.139.5

Luthar SS, Cicchetti D, Becker B (2000) The construct of resilience: a critical evaluation and guidelines for future work. Child Dev 71:543-562

McFarlane CA (2004) Risks associated with the psychological adjustment of humanitarian aid workers. Australas J Disas Trauma. http://www.massey.ac.nz/ trauma/issues/2004-1/mcfarlane.htm

Miller L, Smith AD (1985) August [Wellness Letter]. University of California, Berkeley Archives, Berkeley

National Center for PTSD (2016) PTSD: National center for PTSD. http://www.ptsd. va.gov/PTSD/public/treatment/therapy-med/index.asp

Nolty AAT, Bosch DS, An E, Clements CT, Buckwalter JG (2018) The Headington Institute Resilience Inventory (HIRI): development and validation for humanitarian aid workers. Int Perspect Psychol 7:35-57. https://doi.org/10.103 7/ipp0000080

Panter-Brick C (2021) Systemic resilience and peacebuilding in humanitarian crises. In M. Ungar (ed) . Multisystemic resilience: adaptation and transformation in contexts of change. Oxford University. In press.

Pooley JA, Cohen L, O'Connor M, Taylor M (2013) Posttraumatic stress and posttraumatic growth and their relationship to coping and self-efficacy in Northwest Australian cyclone communities. Psychol Trauma-US 5:392-399. https://doi.org/10.1037/a0028046

Putman KM, Lantz II, Townsend CL, Gallegos AM, Potts AA, Roberts RC, Cree ER, de Villagran M, Eriksson CB, Foy DW (2009) Exposure to violence, support needs, adjustment, and motivators among Guatemalan humanitarian aid workers. Am J Com Psychol 44:109-115. https:/doi.org/10.1007/s10464-009-9249-5

Scholz U, Doña BG, Sud S, Schwarzer R (2002) Is general self-efficacy a universal construct? Psychometric findings from 25 countries. Eur J Psychol Assess 18 : 242-251. https://doi.org/10.1027//1015-5759.18.3.242

Schwarzer R, Born A (1997) Optimistic self-beliefs: assessment of general perceived self-efficacy in thirteen cultures. World Psychol 3(1-2):177-190

Schwarzer R, Jerusalem M (1995) Generalized Self-Efficacy scale. In: Weinman J, Wright $\mathrm{S}$, Johnston $\mathrm{M}$ (eds) Measures in health psychology: a user's portfolio. Causal and control beliefs. NFER-NELSON, Windsor, pp 35-37

Simosi M (2012) The moderating role of self-efficacy in the organizational culture-training transfer relationship. Int J Train Dev 16(2):92-106. https://doi. org/10.1111/j.1468-2419.2011.00396.x

Sitzmann T, Ely K (2011) A meta-analysis of self-regulated learning in work-related training and educational attainment: what we know and where we need to go. Psychol Bull 137:421-442. https://doi.org/10.1037/a0022777

Stanhope DS, Pond SB III, Surface EA (2013) Core self-evaluations and training effectiveness: prediction through motivational intervening mechanisms. J Appl Psychol 98:820-831. https://doi.org/10.1037/a0032599

Stoddard A, Harvey P, Czwarno M, Breckenridge M (2019) Aid Worker Security Report 2019, Speakable: addressing sexual violence and gender-based risk in humanitarian aid. Humanitarian Outcomes, June. 
Strohmeier H, Panter-Brick C (2020) Living with transience in high-risk humanitarian spaces: gendered experiences of international staff and policy implications for building resilience. Disasters. https://doi.org.fuller.idm.oclc org/10.1111/disa.12460

Weathers FW, Litz BT, Keane TM, Palmieri PA, Marx BP, Schnurr PP (2013) The PTSD Checklist for DSM-5 (PCL-5). www.ptsd.va.gov

\section{Publisher's Note}

Springer Nature remains neutral with regard to jurisdictional claims in published maps and institutional affiliations.

Submit your manuscript to a SpringerOpen ${ }^{\circ}$ journal and benefit from:

- Convenient online submission

- Rigorous peer review

- Open access: articles freely available online

- High visibility within the field

- Retaining the copyright to your article

Submit your next manuscript at $\boldsymbol{\sim}$ springeropen.com 